\title{
Momentum Dependence of Resonant Inelastic X-Ray Scattering Spectrum in Insulating Cuprates
}

\author{
K. Tsutsui, T. Tohyama, and S. Maekawa \\ Institute for Materials Research, Tohoku University, Sendai 980-8577, Japan
}

(Received 26 May 1999)

\begin{abstract}
The resonant inelastic x-ray scattering spectrum in insulating cuprates is examined by using the exact diagonalization technique on small clusters in the two-dimensional Hubbard model with second and third neighbor hopping terms. When the incident photon energy is tuned near the $\mathrm{Cu} K$ absorption edges, we find that the features of the unoccupied upper Hubbard band can be extracted from the spectrum through an anisotropic momentum dependence. They provide an opportunity for the understanding of the different behavior of hole- and electron-doped superconductors.
\end{abstract}

PACS numbers: 74.25.Jb, 71.10.Fd, 78.70.Ck

Resonant inelastic x-ray scattering (RIXS) is developing very rapidly into a powerful technique to investigate elementary excitations in the strongly correlated electron systems [- [1]. The application of this technique to insulating copper oxides has made it possible to observe an excitation due to a local charge transfer between copper and oxygen [3] and local $d-d$ excitations on copper site 沺. In addition, it has been demonstrated that, by using high resolution experiments [5], the momentumdependent measurement of the charge transfer gap is possible when the incident photon energy $\omega_{i}$ is tuned through $\mathrm{Cu} K$ absorption edge. Thus, the RIXS can be a useful probe to obtain information on the momentum dependence of the elementary excitations.

One of the elementary excitations in the insulating cuprates is the charge-transfer process from the occupied Zhang-Rice singlet band (ZRB) [6] composed of $\mathrm{Cu} 3 d_{x^{2}-y^{2}}$ and $\mathrm{O} 2 p_{\sigma}$ orbitals to the unoccupied upper Hubbard band (UHB). The dispersion of ZRB have been extensively studied by angle-resolved photoemission spectroscopy (ARPES) experiments on the parent compounds of high $T_{c}$ superconductors [7] 9]: A $d$-wave-like dispersion was observed along the $(0, \pi)-(\pi, 0)$ line with the minimum of the binding energy at $(\pi / 2, \pi / 2)[9]$. On the contrary, the dispersion relation and spectral properties of the unoccupied UHB have not been examined and thus remain to be understood. The information of UHB is of crucial importance for the understanding of the motion of electrons in the electron-doped superconductors. In addition, it may be useful to know if the particle-hole symmetry is required for the high temperature superconductivity.

In this Letter, we examine the RIXS spectrum for the $\mathrm{Cu} \mathrm{K}$-edge, and demonstrate that the characteristic features of the dispersion of UHB can be extracted from the momentum dependence of the spectrum. To see this, we use the half-filled single-band Hubbard model to describe the occupied ZRB and unoccupied UHB by mapping ZRB onto the lower Hubbard band (LHB) in the model. Then, we incorporate $\mathrm{Cu} 1 s$ and $4 p$ orbitals into the model to include the $1 s$-core hole and excited $4 p$ elec- tron into the intermediate state of the RIXS process. The long-range hoppings are also introduced in the Hubbard model with realistic values obtained from the analysis of ARPES data. We find a characteristic momentum dependence of the $\mathrm{Cu} K$-edge RIXS spectrum: The energy of the threshold of the RIXS spectrum at $(\pi / 2, \pi / 2)$ is higher than that at $(0,0)$, whereas the energy of the threshold at $(\pi / 2,0)$ is lower than that at $(0,0)$. This anisotropic dependence is explained by the dispersion of the UHB which has the minimum energy at $(\pi, 0)$ due to the long-range hoppings. The determination of the UHB will contribute to the understanding of the different behavior of hole- and electron-doped superconductors [8, 10].

We map the ZRB onto the LHB, which is equivalent to the elimination of O $2 p$ orbitals. Such mapping was used in the analysis of $\mathrm{O} 1 s$ x-ray absorption spectrum [11]. The Hubbard Hamiltonian with second and third neighbor hoppings for the $3 d$ electron system is written as,

$$
\begin{aligned}
H_{3 d}= & -t \sum_{\langle\mathbf{i}, \mathbf{j}\rangle_{1 \mathrm{st}}, \sigma} d_{\mathbf{i}, \sigma}^{\dagger} d_{\mathbf{j}, \sigma}-t^{\prime} \sum_{\langle\mathbf{i}, \mathbf{j}\rangle_{2 \mathrm{nd}}, \sigma} d_{\mathbf{i}, \sigma}^{\dagger} d_{\mathbf{j}, \sigma} \\
& -t^{\prime \prime} \sum_{\langle\mathbf{i}, \mathbf{j}\rangle_{3 \mathrm{rd}}, \sigma} d_{\mathbf{i}, \sigma}^{\dagger} d_{\mathbf{j}, \sigma}+\text { H.c. }+U \sum_{\mathbf{i}} n_{\mathbf{i}, \uparrow}^{d} n_{\mathbf{i}, \downarrow}^{d},
\end{aligned}
$$

where $d_{\mathbf{i}, \sigma}^{\dagger}$ is the creation operator of $3 d$ electron with spin $\sigma$ at site $\mathbf{i}, n_{\mathbf{i}, \sigma}^{d}=d_{\mathbf{i}, \sigma}^{\dagger} d_{\mathbf{j}, \sigma}$, the summations $\langle\mathbf{i}, \mathbf{j}\rangle_{1 \text { st }}$, $\langle\mathbf{i}, \mathbf{j}\rangle_{2 \text { nd }}$, and $\langle\mathbf{i}, \mathbf{j}\rangle_{3 \text { rd }}$ run over first, second, and third nearest-neighbor pairs, respectively, and the rest of the notation is standard.

Figure 1 shows the schematic process of $\mathrm{Cu} K$-edge RIXS. An absorption of an incident photon with energy $\omega_{i}$, momentum $\mathbf{K}_{i}$, and polarization $\epsilon_{i}$ brings about the dipole transition of an electron from $\mathrm{Cu} 1 s$ to $4 p$ orbital

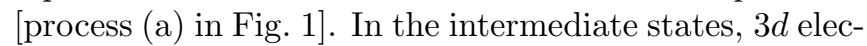
trons interact with a $1 s$-core hole and a photo-excited $4 p$ electron via the Coulomb interactions so that the excitations in the $3 d$ electron system are evolved [process (b)]. The $4 p$ electron goes back to the $1 s$ orbital again and a photon with energy $\omega_{f}$, momentum $\mathbf{K}_{f}$, and polarization 


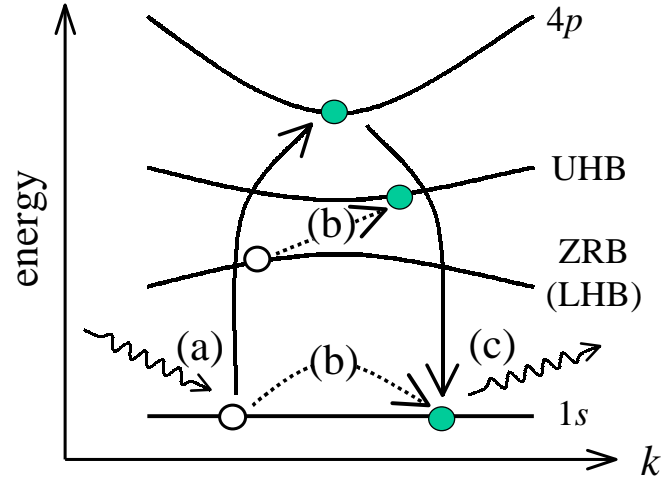

FIG. 1. Schematic picture of the Cu K-edge RIXS process. An incident photon is absorbed and dipole transition $1 s \rightarrow 4 p$ is brought about [process (a)], and through the intermediate state [process (b)], the photo-excited Hpigeldctrontsgives db $1 \mathrm{~s}$ again and a photon is emitted [process (c)].

$\epsilon_{f}$ is emitted [process (c)]. The differences of the energies and the momenta between incident and emitted photons are transferred to the $3 d$ electron system.

In the intermediate state, there are a $1 s$-core hole and a $4 p$ electron, with which $3 d$ electrons interact. Since the $1 s$-core hole is localized because of a small radius of the $\mathrm{Cu} 1 s$ orbital, the attractive interaction between the $1 s$ core hole and $3 d$ electrons is very strong. The interaction is written as,

$$
H_{1 s-3 d}=-V \sum_{\mathbf{i}, \sigma, \sigma^{\prime}} n_{\mathbf{i}, \sigma}^{d} n_{\mathbf{i}, \sigma^{\prime}}^{s},
$$

where $n_{\mathbf{i}, \sigma}^{s}$ is the number operator of $1 s$-core hole with spin $\sigma$ at site $\mathbf{i}$, and $V$ is taken to be positive. On the contrary, since the $4 p$ electron is delocalized, the repulsive interaction between the $4 p$ and $3 d$ electrons as well as the attractive one between the $4 p$ electron and the $1 s$ core hole is small as compared with the $1 s$ - $3 d$ interaction. In addition, when the core-hole is screened by the $3 d$ electrons through the strong $1 s-3 d$ interaction, effective charge that acts on the $4 p$ electron at the core-hole site becomes small. Therefore, the interactions related to the $4 p$ electron are neglected for simplicity. Furthermore, we assume that the photo-excited $4 p$ electron enters into the bottom of the $4 p_{z}$ band with momentum $\mathbf{k}_{0}$, where $z$-axis is perpendicular to the $\mathrm{CuO}_{2}$ plane. This assumption is justified as long as the Coulomb interactions associated with the $4 p$ electron are neglected and the resonance condition is set to the threshold of the $1 s \rightarrow 4 p_{z}$ absorption spectrum 12]. Under these assumptions, the RIXS spectrum is expressed as,

$$
\begin{aligned}
I(\Delta \mathbf{K}, \Delta \omega)= & \sum_{\alpha} \mid\langle\alpha| \sum_{\sigma} s_{\mathbf{k}_{0}-\mathbf{K}_{f}, \sigma} p_{\mathbf{k}_{0}, \sigma} \\
& \times\left.\frac{1}{H-E_{0}-\omega_{i}-i \Gamma} p_{\mathbf{k}_{0}, \sigma}^{\dagger} s_{\mathbf{k}_{0}-\mathbf{K}_{i}, \sigma}^{\dagger}|0\rangle\right|^{2} \\
& \times \delta\left(\Delta \omega-E_{\alpha}+E_{0}\right)
\end{aligned}
$$

where $H=H_{3 d}+H_{1 s-3 d}+H_{1 s, 4 p}, H_{1 s, 4 p}$ being kinetic and on-site energy terms for a $1 s$-core hole and a $4 p$ electron, $\Delta \mathbf{K}=\mathbf{K}_{i}-\mathbf{K}_{f}, \Delta \omega=\omega_{i}-\omega_{f}, s_{\mathbf{k}, \sigma}^{\dagger}\left(p_{\mathbf{k}, \sigma}^{\dagger}\right)$ is the creation operator of the $1 s$-core hole ( $4 p$ electron) with momentum $\mathbf{k}$ and spin $\sigma,|0\rangle$ is the ground state of the half-filled system with energy $E_{0},|\alpha\rangle$ is the final state of the RIXS process with energy $E_{\alpha}$, and $\Gamma$ is the inverse of the relaxation time in the intermediate state. In Eq. (3), the terms $H_{1 s, 4 p}$ are replaced by $\varepsilon_{1 s-4 p}$ which is the energy difference between the $1 s$ level and the bottom of the $4 p_{z}$ band.

The RIXS spectrum of Eq. (3) is calculated on $(\sqrt{8} \times \sqrt{8})-,(\sqrt{10} \times \sqrt{10})-$, and $(4 \times 4)$-site clusters with periodic boundary condition by using a modified version of the conjugate-gradient method together with the Lanczös technique. We will show the results for the $4 \times 4$ site cluster in the following.

The values of the parameters are as follows: $t^{\prime} / t=$ $-0.34, t^{\prime \prime} / t=0.23, U / t=10, V / t=15$, and $\Gamma / t=1$ with $t=0.35 \mathrm{eV}$. The values of $t, t^{\prime}$, and $t^{\prime \prime}$ are the same as those used in the analysis of ARPES data of the high $T_{c}$ superconductors based on the $t-t^{\prime}-t^{\prime \prime}-J$ model [8, 13 . The value of $U$ is obtained from the relation $J=4 t^{2} / U$ and $J / t=0.4$. The value of $V$ is set to be larger than that of $U$. The results shown below are insensitive to the magnitude of $V$ as well as of $\Gamma$ 14].

Before going into the RIXS spectrum, we mention the resonance condition in RIXS. To determine the condition, we have to examine the $\mathrm{Cu} 1 s$ x-ray absorption spectroscopy (XAS) spectrum defined as,

$$
\begin{aligned}
D(\omega)= & \frac{1}{\pi} \operatorname{Im}\langle 0| s_{\mathbf{k}_{0}-\mathbf{K}_{i}, \sigma} p_{\mathbf{k}_{0}, \sigma} \frac{1}{H-E_{0}-\omega-i \Gamma_{\mathrm{XAS}}} \\
& \times p_{\mathbf{k}_{0}, \sigma}^{\dagger} s_{\mathbf{k}_{0}-\mathbf{K}_{i}, \sigma}^{\dagger}|0\rangle,
\end{aligned}
$$

where $H$ is the same as that in Eq. (3). It is necessary to tune the incident photon energy $\omega_{i}$ to the energy region where the $\mathrm{Cu} 1 s$ XAS spectrum appears. The inset in Fig. 2 shows $D(\omega)$, where a two-peak structure appears, i.e., the broad one around $\omega-\varepsilon_{1 s-4 p}=-20 t$ and the sharp one around $-13 t$. The former mainly contains configurations that the core-hole site is doubly occupied by the $3 d$ electrons $(U-2 V=-20 t)$, while the latter dominantly contains configurations that the core-hole site is singly occupied $(-V=-15 t)$. This means that, when the incident energy is tuned around the former structure, the information about UHB can be extracted from the RIXS spectrum. Thus, we set $\omega_{i}$ to the threshold of the XAS spectrum denoted by the arrow in the inset.

Figure 2 shows the momentum dependence of the RIXS spectrum. The spectra below $\Delta \omega / t \sim 2$ and above $\Delta \omega / t \sim 5$ have different origins: The former comes from the excitations related to the spin degree of freedom such as two-magnon Raman scattering, the energy scale of which is so small that the spectrum is hard to be observed [5]. On the other hand, the latter is related to the 


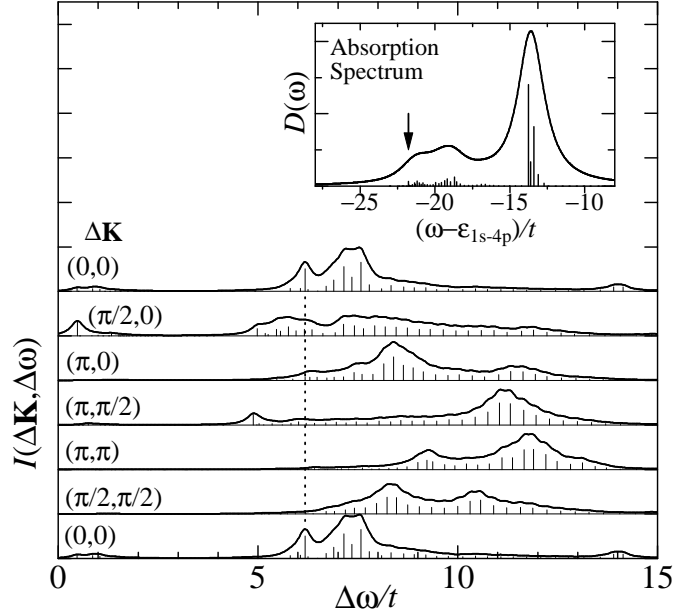

FIG. 2. Resonant inelastic x-ray scattering spectra for $\mathrm{Cu} K$-edge in half-filled Hubbard model with long-range hoppings. The spectra of the elastic scattering process at $\Delta \mathbf{K}=(0,0)$ are not shown. The parameters used are $U / t=10$, $V / t=15, \Gamma / t=1, t^{\prime} / t=-0.34$, and $t^{\prime \prime} / t=F_{10} 023$ Tsthe Yertical dotted line denotes the position of the peak at $\Delta \mathbf{K}=(0,0)$ for guide to eyes. The $\delta$-functions (the vertical thin solid lines) are convoluted with a Lorentzian broadening of $0.2 t$. Inset is the $\mathrm{Cu} 1 \mathrm{~s}$ absorption spectrum with $\Gamma_{\mathrm{XAS}} / t=\Gamma / t=1.0$, and the incident photon energy $\omega_{i}$ is set to the value denoted by the arrow.

excitations from LHB to UHB. The vertical dotted line in the figure denotes the position of the low-energy peak at $\Delta \mathbf{K}=(0,0)$ for guide to eyes. The spectra strongly depend on the momentum showing a feature that the weight shifts to higher energy region with increasing $|\Delta \mathbf{K}|$. This momentum dependence is also obtained in the 10-site cluster calculations. In addition, the threshold of the spectrum at $\Delta \mathbf{K}=(\pi / 2,0)$ and $(\pi, \pi / 2)$ is lower in energy than that at $(0,0)$. At $\Delta \mathbf{K}=(\pi / 2, \pi / 2)$, however, the spectrum appears above the threshold at $(0,0)$, resulting in an anisotropic momentum dependence between the spectra along $(0,0)$ to $(\pi / 2,0)$ and along $(0,0)$ to $(\pi / 2, \pi / 2)$. We note that such an anisotropic feature can not be observed in the RIXS spectrum of the Hubbard model without $t^{\prime}$ and $t^{\prime \prime}$ (not shown) 15. In the 8 -site cluster the spectrum at $\Delta \mathbf{K}=(\pi / 2, \pi / 2)$ is lower in energy than that at $(\pi, 0)$, which is consistent with the above feature.

In the intermediate state, the excitations of the $3 d$ electrons from the occupied to unoccupied states are caused by the interaction with the $1 s$-core hole, i.e., $H_{1 s-3 d}=$ $-V / N \sum_{\mathbf{k}_{1} \sim \mathbf{k}_{4}, \sigma, \sigma^{\prime}} \delta_{\mathbf{k}_{2}-\mathbf{k}_{1}, \mathbf{k}_{3}-\mathbf{k}_{4}} d_{\mathbf{k}_{1}, \sigma}^{\dagger} d_{\mathbf{k}_{2}, \sigma} s_{\mathbf{k}_{3}, \sigma^{\prime}}^{\dagger} s_{\mathbf{k}_{4}, \sigma^{\prime}}$.

The operator $d_{\mathbf{k}_{1}, \sigma}^{\dagger} d_{\mathbf{k}_{2}, \sigma}$ represents a particle-hole excitation. Therefore, we analyze the RIXS process by decomposing into such a particle-hole excitation in order to understand the anisotropic momentum dependence of the threshold along $(0,0)$ to $(\pi / 2,0)$ and along $(0,0)$ to $(\pi / 2, \pi / 2)$ in Fig. 2 .

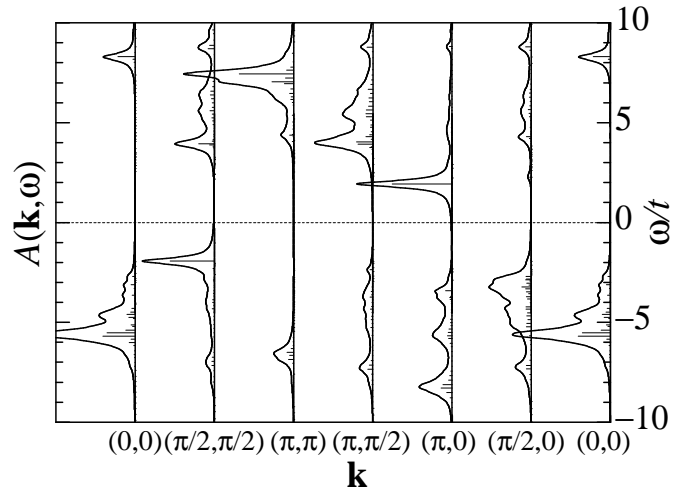

FIG. 3. Single particle excitation spectrum $A(\mathbf{k}, \omega)$ in the half-filled Hubbard model with $t^{\prime}$ and $t^{\prime \prime}$ terms. $t_{\text {Pig. }} t^{\prime}$, $t_{\text {sutsui } e}=\overline{a l}$ al 0.34 and $t^{\prime \prime} / t=0.23$. The dotted line denotes the chemical potential. The $\delta$ functions are convoluted with a Lorentzian broadening of $0.2 t$.

As a first step, we consider the particle-hole excitation as the convolution of the single-particle excitation spectra $A(\mathbf{k}, \omega)$ between occupied LHB and unoccupied UHB. Figure 3 shows $A(\mathbf{k}, \omega)$ in the half-filled Hubbard model with $t^{\prime}$ and $t^{\prime \prime}$ terms $[16]$. Below the chemical potential denoted by the dotted line, a sharp peak appears at $(\pi / 2, \pi / 2)$ with the lowest-binding energy. In contrast, the spectrum at $(\pi, 0)$ is very broad and deep in energy. These features are consistent with the ARPES data for $\mathrm{Sr}_{2} \mathrm{CuO}_{2} \mathrm{Cl}_{2}$ [7, 8 . 3 . Above the chemical potential, the dispersion of UHB has the minimum of the energy at $\mathbf{k}=(\pi, 0)$ 17. We show below that the $(\pi, 0)$ spectrum in UHB plays a crucial role in the RIXS spectrum with $\Delta \mathbf{K}=(\pi / 2,0)$.

Now, we examine the lowest-energy excitations with $\Delta \mathbf{K}=(0,0),(\pi / 2,0)$, and $(\pi / 2, \pi / 2)$ in the convoluted spectrum $\int_{\mathcal{E}<\mu} d \mathcal{E} A(\mathbf{k}+\Delta \mathbf{K}, \mathcal{E}+\omega) A(\mathbf{k}, \mathcal{E})$, where $\mu$ is the chemical potential. For the case that $\Delta \mathbf{K}=(0,0)$, the minimum excitation energy in the convoluted spectrum is $\sim 5 t$ at $\mathbf{k}=(\pi, 0)$. In the same way, the lowest-energy excitation with $\Delta \mathbf{K}=(\pi / 2,0)$ in the convoluted spectrum is that from $\mathbf{k}=(\pi / 2,0)$ of LHB to $(\pi, 0)$ of UHB with the energy of $\sim 4 t$. This value is smaller than that for the $\Delta \mathbf{K}=(0,0)$ case, being consistent with the relation of the thresholds of the RIXS spectra between $\Delta \mathbf{K}=(0,0)$ and $(\pi / 2,0)$. In contrast, the lowest-energy excitation with $\Delta \mathbf{K}=(\pi / 2, \pi / 2)$ in the convoluted spectrum is inconsistent with that in the RIXS spectrum, because the excitation energy, which is determined by the peaks at $\mathbf{k}=(\pi / 2,-\pi / 2)$ of LHB and at $(\pi, 0)$ of UHB, is almost the same as that for $\Delta \mathbf{K}=(\pi / 2,0)$. This means that the argument based on the convoluted spectrum of $A(\mathbf{k}, \omega)$ is insufficient to understand the anisotropic behavior of the threshold, and thus we need to treat the process of the particle-hole excitation exactly. Therefore, we introduce the spectral function $B(\mathbf{k}, \Delta \mathbf{K} ; \omega)$ of the two-body Green's function, which can describe the particle-hole ex- 


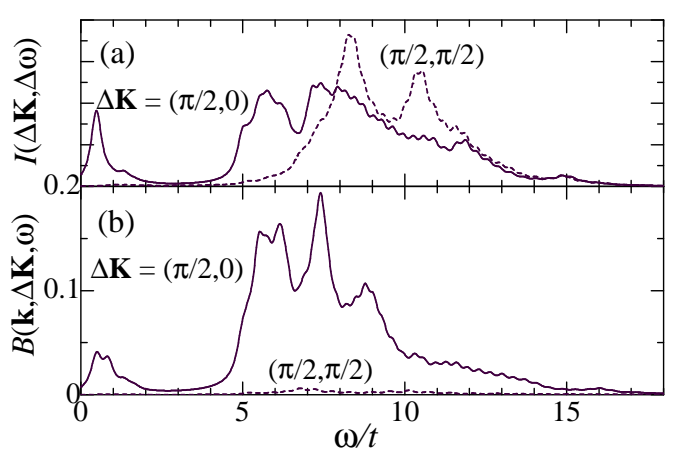

FIG. 4. Spectral function $B(\mathbf{k}, \Delta \mathbf{K}, \omega)$ which represents the particle-hole excitation process Fi(lokerequi $e^{2 t}$ panel), with $[\mathbf{k}, \Delta \mathbf{K}]=[(\pi / 2,0),(\pi / 2,0)]$ (solid line) and $[(\pi / 2,-\pi / 2),(\pi / 2, \pi / 2)]$ (dotted line). Upper panel shows the corresponding RIXS spectra $I(\Delta \mathbf{K}, \Delta \omega)$ which are given in Fig. 2.

citation, defined as

$$
\begin{aligned}
B(\mathbf{k}, \Delta \mathbf{K} ; \omega)= & \sum_{\alpha}\left|\left\langle\alpha\left|\sum_{\sigma} d_{\mathbf{k}+\Delta \mathbf{K}, \sigma}^{\dagger} d_{\mathbf{k}, \sigma}\right| 0\right\rangle\right|^{2} \\
& \times \delta\left(\omega-E_{\alpha}+E_{0}\right),
\end{aligned}
$$

where the states $|\alpha\rangle$ have the same point-group symmetry as that of the final states of the RIXS process. Figure shows $B(\mathbf{k}, \Delta \mathbf{K} ; \omega)$ with $[\mathbf{k}, \Delta \mathbf{K}]=[(\pi / 2,0),(\pi / 2,0)]$ and $[(\pi / 2,-\pi / 2),(\pi / 2, \pi / 2)]$. Note that $\mathbf{k}+\Delta \mathbf{K}=$ $(\pi, 0)$ in both cases. The spectrum with $[\mathbf{k}, \Delta \mathbf{K}]=$ $[(\pi / 2,0),(\pi / 2,0)]$ reproduces very well the RIXS spectrum near the threshold. The intensity of $B(\mathbf{k}, \Delta \mathbf{K} ; \omega)$ with $\Delta \mathbf{K}=(\pi / 2, \pi / 2)$ is very small, implying that the process $\sum_{\sigma} d_{(\pi, 0), \sigma}^{\dagger} d_{(\pi / 2,-\pi / 2), \sigma}$ is almost forbidden [18]. This is the origin of the small weight of $I(\Delta \mathbf{K}, \omega)$ with $\Delta \mathbf{K}=(\pi / 2, \pi / 2)$ around $\omega / t \sim 5$. We note that this behavior can not be obtained by the convolution of $A(\mathbf{k}, \omega)$ mentioned above. At the higher energy region, $\omega / t \sim 7$, different processes keeping $\Delta \mathbf{K}=(\pi / 2, \pi / 2)$, for example, the annihilation of the $(\pi / 2,0)$ electron and the creation of the $(\pi, \pi / 2)$ one, dominate the excitations. These processes induce the large weight above $\omega / t \sim 7$.

In summary, we have examined the momentum dependence of the $\mathrm{Cu} K$-edge RIXS spectrum by using numerically exact diagonalization technique on small clusters. Regarding the ZRB as the LHB, we have adopted the Hubbard model with $\mathrm{Cu} 1 s$ - and $4 p$-bands. We have also introduced the long-range hoppings, $t^{\prime}$ and $t^{\prime \prime}$, of the ZR singlet, and found that the threshold of the spectrum at $\Delta \mathbf{K}=(\pi / 2,0)$ is small compared with that at $(0,0)$, whereas the threshold at $(\pi / 2, \pi / 2)$ is larger than that at $(0,0)$. This dependence is caused by the $(\pi, 0)$ state in UHB. Thus, by examining the RIXS spectrum, we can extract the property of the unoccupied states that is crucially important for the electron-doped superconductors and also for the understanding of the behavior different from the hole-doped superconductors [8,10]. Very recently, Stanford's group has reported [19] that interesting data with momentum dependent inelastic scattering signal has been seen in the $2 \mathrm{eV}$ range, which reveals the property of unoccupied states right above the charge-transfer gap. These progress in both theory and experiment will open a new prospect of the physics of cuprates.

The authors thank Z.-X. Shen for valuable discussions. This work was supported by Priority-Areas Grants from the Ministry of Education, Science, Culture and Sport of Japan, CREST, and NEDO. Computations were carried out in ISSP, Univ. of Tokyo; IMR, Tohoku Univ.; and Tohoku Univ..

[1] C.-C. Kao et al., Phys. Rev. B 54, 16361 (1996).

[2] S. M. Butorin et al., Phys. Rev. B 55, 4242 (1997).

[3] J. P. Hill et al., Phys. Rev. Lett. 80, 4967 (1998).

[4] P. Kuiper et al., Phys. Rev. Lett. 80, 5204 (1998).

[5] P. Abbamonte et al., cond-mat/9810095. The best resolution used was $0.45 \mathrm{eV}$.

[6] F. C. Zhang and T. M. Rice, Phys. Rev. B 37, 3759 (1988).

[7] B. O. Wells et al., Phys. Rev. Lett. 74, 964 (1995).

[8] C. Kim et al., Phys. Rev. Lett. 80, 4245 (1998).

[9] F. Ronning et al., Science, 282, 2067 (1998).

[10] H. Takagi et al., Physica C 162-164, 1001 (1989).

[11] C. T. Chen et al., Phys. Rev. Lett. 66, 104 (1991).

[12] The experimental data for the $\mathrm{Cu} K$-edge absorption spectrum show that the out-of-plane $(z)$ polarized peaks are lower in energy than in-plane polarized peaks [20]. In order to examine the RIXS spectrum with the single component of the $4 p$ bands, we consider the case of $z$-polarized photon.

[13] In 8- and 10-site clusters, only $t$ and $t^{\prime}$ are included for hopping parameters due to their small system sizes.

[14] P. M. Platzman and E. D. Isaacs, Phys. Rev. B 57, 11107 (1998).

[15] K. Tsutsui, T. Tohyama, and S. Maekawa (unpublished).

[16] For $A(\mathbf{k}, \omega)$ in the Hubbard model without $t^{\prime}$ and $t^{\prime \prime}$, see P. W. Leung et al., Phys. Rev. B 46, 11779 (1992).

[17] In the large $U$ limit, the dispersion of the UHB is described by the single-hole dispersion of the $t-t^{\prime}-t^{\prime \prime}-J$ model with $t<0, t^{\prime}>0$, and $t^{\prime \prime}<0$. The sign difference comes from the fact that the carrier in UHB is an electron [see T. Tohyama. and S. Maekawa. Phys. Rev. B 49, 3596 (1994)]. Although the energy at $(\pi, 0)$ is almost the same as that at $(\pi / 2, \pi / 2)$ in the $t-J$ model, the former decreases when $t^{\prime}(>0)$ and $t^{\prime \prime}(<0)$ are introduced. By defining $\epsilon(\mathbf{k})=4 t^{\prime} \cos k_{x} \cos k_{y}+2 t^{\prime \prime}\left(\cos k_{x}+\cos k_{y}\right)$, the energy difference between at $(\pi / 2, \pi / 2)$ and $(\pi, 0)$ is proportional to $\epsilon(\pi / 2, \pi / 2)-\epsilon(\pi, 0)=-8 t^{\prime \prime}+4 t^{\prime}$.

[18] In the spin-density wave mean-field approximation, $B(\mathbf{k}, \Delta \mathbf{K} ; \omega)$ with $[\mathbf{k}, \Delta \mathbf{K}]=[(\pi / 2,-\pi / 2),(\pi / 2, \pi / 2)]$ is rigorously zero. This is due to the effect of the coherence factor arising from the antiferromagnetic long-range order as is the case of the BCS superconductivity. The detail will be shown elsewhere.

[19] Z. Hasan, E. Issacs, and Z.-X. Shen (unpublished).

[20] N. Kosugi et al., Chem. Phys. 135, 149 (1989) 